\title{
Vorwort Heft 1-2011
}

\author{
Hans-Christoph Grunau
}

Online publiziert: 18. Dezember 2010

(C) Vieweg+Teubner und Deutsche Mathematiker-Vereinigung 2010

Am 21. März 2010 verstarb Fritz Grunewald vollkommen unerwartet im Alter von nur 60 Jahren. Angehörige, Freunde, Kollegen, Schüler waren schockiert und traurig. Der Tod dieses wichtigen Vertreters der Geometrie und der Gruppen- und Zahlentheorie war vielleicht auch deswegen für so Viele so bewegend, da Fritz Grunewald intensiv mit außerordentlich vielen Kolleginnen und Kollegen zusammengearbeitet hat. Nahezu alle seiner mehr als 100 mathematischen Arbeiten sind in Zusammenarbeit mit insgesamt 48 Koautorinnen und Koautoren entstanden. Den Autor Dan Segal des vorliegenden Nachrufs verbinden eine 37-jährige Freundschaft und 20 gemeinsame Publikationen mit Fritz Grunewald. Das englische Original dieses Beitrags, der von Gabriele Nebe ins Deutsche übersetzt wurde, wird im Bulletin of the London Mathematical Society erscheinen. Für deren Einverständnis mit der Publikation einer deutschen Version ist der Jahresbericht sehr dankbar.

Der zweite Beitrag im vorliegenden Heft ist ein Übersichtsartikel über „Random networks with concave preferential attachment rule“ von Steffen Dereich and Peter Mörters. Netzwerke sind überall zu beobachten; man kann sowohl an soziale Netzwerke, die mathematics genealogy oder an das world wide web denken. Eine zufällige und im Artikel genauer spezifizierte ,konkave“ Regel für das Hinzufügen neuer Knoten und Kanten zugrundelegend studieren die Autoren typische Eigenschaften der daraus resultierenden zufälligen Netzwerke. Diese betreffen etwa die Beobachtungen, dass es meist sehr wenige ausgezeichnete Knoten gibt, die mit sehr vielen anderen Knoten verbunden sind oder dass meist der Abstand zwischen verschiedenen Knoten relativ gering ist.

H.-Ch. Grunau (凶)

Institut für Analysis und Numerik, Fakultät für Mathematik, Otto-von-Guericke-Universität, Postfach 4120, 39016 Magdeburg, Deutschland

e-mail: hans-christoph.grunau@ovgu.de 
Die Buchbesprechungen nehmen in diesem Heft einen recht breiten Raum ein und widmen sich Neuerscheinungen aus den Themenbereichen: Mathematische Biologie, Ricci-Fluss und der Beweis des differenzierbaren $\frac{1}{4}$-pinching-Theorems, sowie angewandte harmonische Analysis.

Nachdem die online-Präsenz des Jahresberichts auf den DMV-Internetseiten - bedingt durch einen software-update gab es vorübergehend Einschränkungen - nun wieder voll funktionsfähig ist, wollen wir einen ersten Schritt in eine interaktive Richtung gehen. Auf den DMV-Seiten finden Sie nun auch ein Forum für Leserinnen und Leser, in denen Sie sich im Internet zum Jahresbericht äußern können: Möchten Sie Kritik oder Anregungen mitteilen? Welche Themen haben Sie besonders angesprochen? Haben Sie Vorschläge, welche Bücher besprochen werden sollten?

Im Moment sind die „Mathemacher“ schreibberechtigt, mittelfristig soll dieses auf die DMV-Mitglieder umgestellt werden. Nachdem Sie sich registriert haben, können Sie sich nach Anklicken von „Publikationen $\rightarrow$ Jahresbericht der DMV $\rightarrow$ Leserforum“ auf den DMV-Internetseiten anmelden und Beiträge hinzufügen. Einen ersten Beitrag habe ich schon verfasst, auf Ihre Reaktionen und weitere Meinungsäußerungen sind die Herausgeber sehr gespannt! 